\title{
Monocyte-Platelet Complexes in Myocardial Infarction: Sub-Sets and Platelet-Derived Microvesicles Matter
}

\author{
Lina Badimon ${ }^{1}$ \\ ${ }^{1}$ Cardiovascular Program-ICCC, IR-Hospital de la Santa Creu i Sant Pau, \\ Barcelona, Spain
}

Thromb Haemost 2018;118:1854-1855.

Atherosclerosis is a chronic lipid-driven inflammatory disease of the arterial wall characterized by the involvement of the innate and adaptive immune system. ${ }^{1-3}$ Low-density lipoproteins enhance a series of pro-inflammatory reactions perpetuating the activation, recruitment and transmigration of different innate immune cells (monocytes, mast cells, neutrophils, natural killer cells and dendritic cells). Although the contribution of circulating monocytes is essential, acquired immunity, mainly performed by $\mathrm{T}$ cells (Th1- and Th2), is also critically involved in atherosclerosis lesion progression. Monocytes and macrophages are very versatile, and depending on the local micro-environment they can assume different phenotypes and functional characteristics, a termed referred to as 'polarization' (a reversible process). ${ }^{4,5}$

Platelets are released into the circulation as cytoplasmic fragments of bone marrow megakaryocytes and circulate in the blood stream for 7 to 10 days without interacting with other blood elements and/or the vascular wall. Upon endothelial injury or rupture of an atherosclerotic plaque, platelets become activated and anchor on the damage vessel wall. ${ }^{6}$ Rapid platelet recruitment induces thrombosis and target organ ischaemia. In the heart, atherothrombosis induce acute coronary syndromes (ACSs). The initial platelet tethering is mainly mediated by the glycoprotein Ib alpha (GPIb $\alpha$ ) receptor (the main binding region of GPIb/IX/V platelet complex) that also contains binding sites for leukocyte integrin macrophage- 1 antigen and P-selectin, which favours further platelets and leukocyte recruitment. ${ }^{6}$ Activated monocytes can form complexes with activated platelets by specific interaction of P-selectin on activated platelets with P-selectin glycoprotein ligand-1, which is expressed by monocytes and further stabilized by additional integrin adhesion between these cells. In 1991, the dynamics of leukocyte-platelet adhesion and platelet-platelet interaction in the whole blood were first described and since then
Address for correspondence Lina Badimon, PhD, Cardiovascular Program-ICCC, IR-Hospital de la Santa Creu i Sant Pau, Barcelona, Spain (e-mail: Ibadimon@santpau.cat).

the impact of these interactions in ACS has been analysed in different studies. ${ }^{7}$ More recently, increased platelet-monocyte counts have been associated with different monocyte sub-sets and levels of activation. In this issue, an interesting paper by Elena Vasilieva's group investigated not only platelet-monocyte and monocyte sub-set interactions in acute myocardial infarction but also how platelet-derived microvesicles participate in these interactions. ${ }^{8}$ Indeed, plateletderived extracellular vesicles have been demonstrated to contribute to thrombosis and be markers of active disease in different types of patients even when patients were treated as per guidelines. ${ }^{9,10}$

Conflict of Interest

None.

\section{References}

1 Badimon L, Storey RF, Vilahur G. Update on lipids, inflammation and atherothrombosis. Thromb Haemost 2011;105(Suppl 1): S34-S42

2 Hansson GK, Libby P, Schönbeck U, Yan ZQ. Innate and adaptive immunity in the pathogenesis of atherosclerosis. Circ Res 2002;91 (04):281-291

3 Libby P, Nahrendorf M, Swirski FK. Monocyte heterogeneity in cardiovascular disease. Semin Immunopathol 2013;35(05): 553-562

4 Weber C, Shantsila E, Hristov M, et al. Role and analysis of monocyte subsets in cardiovascular disease. Joint consensus document of the European Society of Cardiology (ESC) Working Groups "Atherosclerosis \& Vascular Biology" and "Thrombosis". Thromb Haemost 2016;116(04):626-637

5 Tuñón J, Bäck M, Badimón L, et al; ESC Working Group on Atherosclerosis and Vascular Biology. Interplay between hypercholesterolaemia and inflammation in atherosclerosis: translating experimental targets into clinical practice. Eur J Prev Cardiol 2018;25(09):948-955

6 Badimon L, Vilahur G. Thrombosis formation on atherosclerotic lesions and plaque rupture. J Intern Med 2014;276(06):618-632 received

October 4, 2018

accepted

October 4, 2018 (c) 2018 Georg Thieme Verlag KG Stuttgart · New York
DOI https://doi.org/

$10.1055 / \mathrm{s}-0038-1675242$. ISSN 0340-6245. 
7 Rinder HM, Bonan JL, Rinder CS, Ault KA, Smith BR. Dynamics of leukocyte-platelet adhesion in whole blood. Blood 1991;78(07): 1730-1737

8 Loguinova M, Pinegina N, Kogan V, et al. Monocytes of different subsets in complexes with platelets in patients with myocardial infarction. Thromb Haemost Haemost 2018;118(11):. Doi: 10.1055/ s-0038-1673342
9 Badimon L, Suades R, Arderiu G, Peña E, Chiva-Blanch G, Padró T. Microvesicles in atherosclerosis and angiogenesis: from bench to bedside and reverse. Front Cardiovasc Med 2017;4:77

10 RidgerVC, Boulanger CM, Angelillo-Scherrer A, et al; Position Paper of the European Society of Cardiology (ESC) Working Group on Atherosclerosis and Vascular Biology. Microvesicles in vascular homeostasis and diseases. Thromb Haemost 2017;117(07):1296-1316 